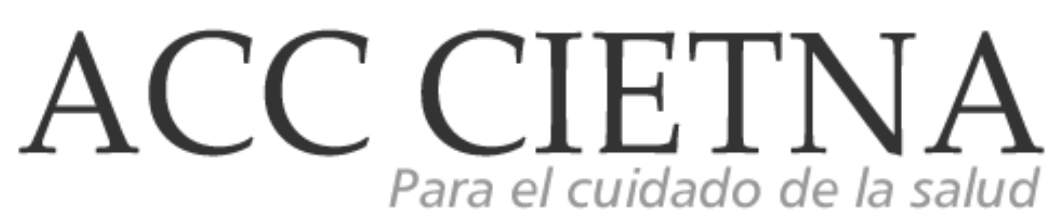

\title{
Experiencias de las enfermeras en el cuidado post operatorio inmediato. Hospital I Naylamp EsSalud - Chiclayo
}

\author{
Morales Huari Regina Soledad ${ }^{1}$, Santamaría Chapoñán Luz Mariela² , Vizconde Campos María \\ Olinda ${ }^{3}$
}

\begin{tabular}{l}
\hline INFORMACIÓN DEL ARTÍCULO RESUMEN \\
\hline Historia del artículo:
\end{tabular}

Recibido el 23 de mayo de 2016

Aceptado el 15 de octubre de 2017

Palabras claves:

Experiencia

Enfermería

Cuidado Post operatorio inmediato

La presente investigación cualitativa, con abordaje fenomenológico tuvo como objetivo describir, analizar y comprender estas experiencias en la Unidad de Cuidados Post anestésicos del Hospital Naylamp EsSalud de Chiclayo. Se sustentó en los conceptos de experiencia (Wojtyla), cuidado (Waldow), post operatorio inmediato (Almeida). Se tuvieron como sujetos de estudio a siete enfermeras, la muestra se determinó por saturación; los datos se recolectaron mediante la entrevista a profundidad no estructurada y se trataron mediante el análisis fenomenológico. Se tuvo en cuenta los criterios de rigor ético según Sgreccia y rigor científico según Hernández. Obteniéndose las siguientes proposiciones: Entre la satisfacción e insatisfacción al brindar cuidados post operatorios. Brindando un cuidado integral a la persona post operada. Responsabilidad vs Temor e impotencia frente a las complicaciones post operatorias.

Conclusión: Las experiencias de las enfermeras en el cuidado post operatorio inmediato de la unidad de cuidados post anestésicos afloran diferentes sentimientos ante la situación que se encuentra la persona post operada brindando cuidados en la parte física, psicológica, emocional y espiritual; además de la comunicación interpersonal con la

\footnotetext{
${ }^{1}$ Licenciada en Enfermería. Centro de Salud Tingo de Ponasa - San Martin, Perú. Email: regina.soledad.m@gmail.com

2 Licenciada. Enfermera con trabajo independiente, Chiclayo, Perú.Email: luzsantamria9133@gmail.com

Docente de la Escuela de Enfermería de la Universidad Católica Santo Toribio de Mogrovejo, Chiclayo, Perú.

Email: mvizconde@usat.edu.pe
} 
persona post operada, enfermera y familia frente a algunas complicaciones en la cual asume responsabilidad y compromiso en la toma de decisiones durante el cuidado.

Experiences of nurses in immediate postoperative care. I Naylamp EsSalud Hospital - Chiclayo

ABSTRACT

\section{Keywords:}

Experience

Nursing care

Immediate postoperative period
This qualitative research with phenomenological approach is as an aimed to describe, analyze and understand these experiences in the Post Anesthetic Care Unit of the Hospital of Chiclayo EsSalud Naylamp. It was based on the concepts of experience (Wojtyla), care (Waldow), immediate postoperative (Almeida). They were taken as subjects to seven nurses, the sample was determined by saturation; Data were collected through unstructured interviews depth and treated by the phenomenological analysis. Rigorous ethical criteria taken into account as Sgreccia and scientific rigor according to Hernandez. Yielding the following propositions: Between satisfaction and dissatisfaction to provide post-operative care. Providing comprehensive care to the person operated. Responsibility vs Fear and powerlessness against postoperative complications. Conclusion: The experiences of nurses in the immediate post-operative care of post anesthetic care unit arise different feelings about the situation that the person providing care post operated in physical, psychological, emotional and spiritual part is; in addition to interpersonal communication with the post person operated, nurse and family face some complications which assumes responsibility and accountability in decision-making in the care.

\section{Introducción}

Hoy en día, enfermería ha ido evolucionando y conforme han transcurrido los años se observa la necesidad e importancia que tiene su labor en el cuidado de la persona, familia y comunidad. Esto motiva a la enfermera a utilizar y aplicar en la práctica el pensamiento crítico para la toma de decisiones adecuadas, con el fin de proteger a la persona de manera holística y brindar cuidados con una visión pandimensional ${ }^{1}$.

Enfermería es la ciencia, el arte de cuidar, la atención autónoma y la asistencia dispensada de la salud del individuo, familia y comunidad. Su campo de acción es la promoción y el mantenimiento de la salud, la prevención de la enfermedad y su participación en el tratamiento, 
incluyendo la rehabilitación, independientemente de la etapa de vida que atraviesa la persona, familia o comunidad. El objetivo de enfermería es mantener al máximo el bienestar físico, mental, social y espiritual del ser humano².

El ser humano es responsable y tiene la capacidad de tomar decisiones en relación a su salud, sin embargo se enfrenta a diferentes situaciones dependiendo de los estilos de vida que profesa, antecedentes familiares y también la edad; como consecuencia de los mismos puede atravesar experiencias quirúrgicas para solucionar determinadas enfermedades, de manera paliativas o restablecer su salud. Una intervención de tipo quirúrgico, es una acción mecánica sobre una estructura anatómica del cuerpo realizada por un cirujano en la cual se acompaña de un anestesiólogo y enfermeras especialistas; las cirugías pueden desarrollarse como parte de un tratamiento para la solución de un problema o con la finalidad de establecer un diagnóstico ${ }^{3}$.

Los profesionales de enfermería desempeñan sus cuidados en las diferentes áreas críticas como son emergencia, $\mathrm{UCl}$ y centro quirúrgico, en el cual se encuentra el área de cuidados post anestésicos, donde la persona se recupera hemodinamicamente de la experiencia de una intervención quirúrgica, en esta unidad pasa las primeras horas post operatorias y post anestésicas en el cuidado de enfermería, en el que está dirigido a prevenir posibles complicaciones producto de los efectos anestésicos, de la intervención quirúrgica hasta el equilibrio y sea trasladado la persona al área de hospitalización donde logrará su total recuperación.

Los estudios relacionados con el cuidado de la persona en el post operatorio inmediato hacen referencia a la forma cómo se busca restablecer el equilibrio fisiológico interrumpido, el alivio del dolor y la prevención de complicaciones durante la estancia de la persona en la unidad de cuidados post anestésicos ${ }^{4}$. Así mismo Burga $^{5}$ sostiene que las enfermeras basan sus cuidados sólo en las indicaciones médicas, olvidándose del ser cuidado y del objeto de enfermería como profesión. Esto lleva a las investigadoras a plantearse interrogantes ¿El cuidado de enfermería, solo se basa en el cumplimiento de las prescripciones médicas? ¿La enfermera es la única que realiza la valoración neurológica inicial al paciente quirúrgico? ¿Cómo es el cuidado de la enfermera en las personas post operadas? ¿El cuidado que brinda involucra todas las dimensiones de la persona?

El cuidado de la enfermera en la unidad de cuidados post anestésicos tiene como propósito identificar la importancia de los signos que manifiesta la persona post operada, anticiparse y prevenir complicaciones post operatorias, además de cubrir sus necesidades y problemas hasta lograr su recuperación por completo de la anestesia. El objetivo en esta etapa es asegurar la conservación de la función respiratoria adecuada, vigilar y prevenir la depresión respiratoria, mantener el estado hemodinámico de la persona, vigilar el estado de conciencia y condiciones generales de la persona post operada, conservar la seguridad y promover su comodidad; también involucran control de signos vitales, vigilar la posición, control del dolor, vigilancia de posibles signos de alerta a nivel cardiovascular, neurológico $y$ gastrointestinal o la aparición de hemorragias y por último e importante registrar el proceso de cuidado en el post operatorio ${ }^{6}$.

A nivel local, en el Hospital I Naylamp EsSalud, se encuentra la unidad de centro quirúrgico, donde se realizan diariamente 8 a 10 cirugías programadas como electivas y de emergencia, en las especialidades de otorrinolaringología, ginecología, oftalmología, cirugía general, urología. Cuando las personas salen de quirófano son recepcionados por el personal de 
recuperación: enfermera y técnico de enfermería en la mayoría de los casos, y sólo en las cirugías de otorrinolaringología participa el médico anestesiólogo. Como es evidente, el profesional de enfermería participa en el cuidado de las personas que ingresan a la unidad de cuidados post anestésicos. Según refieren las enfermeras, las personas post operadas permanecen en recuperación por un periodo promedio de dos a tres horas, luego son hospitalizados o dados de alta según la valoración post anestésica y la evaluación e indicación del médico tratante.

Se ha observado que las enfermeras en la unidad de cuidados post anestésicos realizan varias actividades que ellas mismas expresan de la siguiente manera: “... recepción de la persona, realizo la valoración hemodinámica y el dolor de acuerdo las diferentes escalas, sigo la prescripción médica, si no obtengo resultados de recuperación consulto al médico para administración de otros medicamentos...”,“...aplicamos las escalas de Aldrette, de Bromage y verificamos la evolución de la persona", "... es muy importante el control de los signos vitales, monitorizamos las primeras dos horas cada quince minutos, luego cada treinta minutos, ya que nos dan referencia de cómo está reaccionando el organismo del paciente y así verificar alguna complicación, si se presentan casos se reacciona inmediatamente...".

Las enfermeras hacen referencia a las actividades que permiten la recuperación fisiológica de las personas, el control del dolor y la prevención de complicaciones; pero llama la atención que no se escucha en sus discursos decir "cuidado de enfermería", cuando mencionan su relación con las personas. Ellas refieren:"...yo lo atiendo, monitorizo, aplico las escalas de valoración post anestésicos, administro tratamiento indicado, coordino una cama para que se hospitalice, etc...", omitiendo la relación existencial que pueden establecer con el ser cuidado y si lo realizan no lo expresan.

Además durante las prácticas hospitalarias se ha escuchado a otros profesionales y personal de salud expresar en relación a las enfermeras de la Unidad de Cuidados Post anestésicos: “...las enfermeras sólo recepcionan a las personas post operadas, les controlan las funciones vitales, administran tratamiento, las tienen unas horas, coordinan una cama y lo hospitalizan...". Según carrillo el profesional de enfermería en el post operatorio inmediato, realiza una evaluación del estado general, de acuerdo a una serie de cuidados que se refieren a protocolo de valoración que incluyen valorar la actividad muscular, respiración, circulación, conciencia, coloración de la piel y los signos vitales, puesto que proporcionan una base sólida que permite desarrollar cuidados individualizados y de calidad?

Es por ello ante esta problemática se formula la siguiente pregunta ¿Cuáles son las experiencias de las enfermeras en el cuidado post operatorio inmediato. Hospital I Naylamp EsSalud - Chiclayo 2014? Siendo así el objeto de investigación, la experiencia de las enfermeras en el cuidado post operatorio inmediato. Ya que la presente investigación se realizó con el único objetivo de describir, analizar y comprender las experiencias de las enfermeras en el cuidado post operatorio inmediato.

El presente estudio se justificó porque hoy en día los cuidados de las enfermeras a nivel mundial, nacional y local requiere que desarrollen conocimientos y competencias necesarias para poder brindar un cuidado humanizado, más aún a las personas que se encuentran en el post operatorio inmediato, en la unidad de cuidados post anestésicos, ya que las primeras horas post operatorias son cruciales para su recuperación, la persona presenta muchas veces producto de los efectos anestésicos y de la intervención quirúrgica 
depresión del sistema respiratorio, del sistema nervioso central, desequilibrio hemodinámico, predisponiéndolos a futuras complicaciones en su recuperación por lo que necesitan cuidados y monitorización permanente por el profesional de enfermería, la cual debe contar con conocimientos y destrezas para hacer frente a estos eventos.

Fue importante profundizar y develar las experiencias de las enfermeras en el cuidado de la persona post operada en la unidad de cuidados post anestésicos, cómo enfrenta y expresa sus sentimientos frente a la experiencia del cuidado lo cual pretende describir, analizar y comprender estas experiencias, así como develar su relación profesional con el otro ser es en ese momento transformador, único y propio que vive cada una de ellas, ofreciendo la oportunidad de mejorar en el día a día la práctica del cuidado de enfermería.

Al develar estas experiencias de las enfermeras en el cuidado post operatorio inmediato y reflexionar sobre las mismas, se contribuyó a ampliar el cuerpo de conocimientos de enfermería en esta área de cuidados como es la unidad de recuperación post anestésica, así mismo a proponer estos conocimientos en el plan de estudio de pregrado como parte de los temas de investigación de la asignatura de cuidado al Adulto con Problemas de Menor Complejidad y en la estructura del plan de estudios de la segunda especialidad de enfermería en el centro quirúrgico ya que permitió profundizar en el cuidado $y$ reflexionar sobre las competencias del perfil de la enfermera en esta área, además ser considerados como antecedentes para futuras investigaciones que se relacionen con el objeto de estudio.

\section{Metodología}

El tipo de abordaje que la investigación asumió, tiene perspectiva metodológica cualitativa, bajo el enfoque de estudio fenomenológico ${ }^{8}$, se desarrolló teniendo en cuenta los momentos de la trayectoria fenomenológica: la descripción del fenómeno, la reducción del fenómeno, y la comprensión o interpretación del fenómeno: análisis ideográfico y análisis nomotético ${ }^{9}$.

La muestra delimitada por saturación ${ }^{10}$, fueron siete enfermeras, teniendo como criterio de inclusión: enfermeras con segunda especialidad en centro quirúrgico, como promedio mínimo de 5 años de experiencia o desempeño en el área por el dominio de la misma y mayor tiempo de experiencia profesional en el servicio de la Unidad de Cuidados Post anestésicos y que acepten libremente participar de la investigación. El escenario de investigación fue el Hospital I Naylamp EsSalud - Chiclayo, institución que pertenece al primer nivel de atención. Dentro de los diferentes servicios con los que cuenta dicha institución, está el servicio de Centro Quirúrgico conformado por dos quirófanos y una sala de recuperación donde se brinda atención de las especialidades de ginecología, otorrinolaringología, urología, cirugía y oftalmología; para resolver problemas de salud a través de las cirugías de cesáreas, legrado uterino, apendicetomía, histerectomía, excersion de catarata, trabeculectomía, amigdalotomía, etc. El tipo de anestesia que utilizan es anestesia general, regional y local. Las enfermeras son programadas de forma rotativa en diferentes áreas como sala de operación, sala de recuperación y central de esterilización. Ellas ofrecen cuidado de enfermería en el intraoperatorio y post operatorio inmediato. 
Para la ejecución del estudio fue necesario obtener los permisos respectivos a la institución de salud mediante una solicitud y luego el consentimiento informado a los sujetos que cumplieron los criterios de inclusión, el cual se realizó en el servicio de centro quirúrgico: Unidad de cuidados post anestésicos; las entrevistas fueron transcritas conforme fueron ejecutadas, luego para dar mayor credibilidad y conformidad a los relatos se volvió a visitar a las enfermeras para corroborar lo vertido y profundizar en algunos contenidos. Cabe resaltar que para preservar la identidad de las enfermeras se les asignó un seudónimo de flores, una vez que se saturó los datos se procedió a organizar las entrevistas para el análisis respectivo.

Después de la recolección de información se procedió al análisis de los datos de contenido como el análisis nomotético y procedimiento para la construcción del cuadro nomotético ${ }^{11}$, luego se realizó la clasificación de las unidades obtenidas en proposiciones, estableciendo conexiones entre las mismas contrastando la realidad con otras literaturas y con los puntos de vista de las investigadoras para obtener como resultado final las proposiciones de estudio, relacionadas al objeto y a los objetivos del estudio fenomenológico.

Para el desarrollo de la investigación se tuvo en cuenta los principios de bioética personalista: el valor fundamental de la vida, principio de confidencialidad, principio de libertad $y$ responsabilidad, principio de sociabilidad $y$ subsidiariedad12. Así mismo los siguientes principios de rigor científico: credibilidad, confirmabilidad o auditabilidad ${ }^{13}$.

\section{Resultados, análisis y discusión}

\section{A. ENTRE \\ LA SATISFACCIÓN \\ $E$ \\ INSATISFACCIÓN AL BRINDAR CUIDADOS POST OPERATORIOS.}

Wojtyla ${ }^{14}$ señala que la experiencia es el modo que tenemos de acceder al conocimiento de nuestro mundo y de nosotros mismos. Todo conocimiento que nosotros podamos obtener de las cosas y de otros hombres, parten de la experiencia que se ha tenido directa o indirectamente de nosotros mismos. Pero esta comprensión del hombre que yo o alguien posean, es distinta a la comprensión que yo posea de mí mismo. El yo que percibe no se percibe corporalmente poseyendo un cuerpo sino que va más allá de la mera percepción física y alcanza la corporalidad y sensibilidad de la interioridad subjetiva de la persona.

Por consiguiente un sentimiento es un estado del ánimo que se produce por causas que lo impresionan, y éstas pueden ser alegres, felices, dolorosas y tristes; además surge como resultado de una emoción que permite que el sujeto sea consciente de su estado anímico, en otras palabras, los sentimientos son emociones conceptualizadas que determinan el estado de ánimo. Por tanto, el estado del sujeto es caracterizado por la impresión afectiva que le causa determinada persona, recuerdo o situación en general; por tal motivo cuando éstos son sanos, es posible alcanzar la felicidad y conseguir que la dinámica cerebral fluya con normalidad. En el caso contrario, se experimenta un desequilibrio emocional que puede derivar en el surgimiento de trastornos tales como la depresión ${ }^{15}$.

Los sentimientos se clasifican en: sentimientos sensoriales; que son un tipo especial de sensaciones a pesar que aparecen vinculadas con los sentimientos y tienen un objeto, no son 
intencionales, se caracterizan por poder localizarse en el cuerpo y porque su referencia al yo es indirecta; los sentimientos vitales que a diferencia de los anteriores, estos poseen un carácter difuso porque se extienden a todo el cuerpo y no se reducen a una determinada región del mismo; sentimientos anímicos o psíquicos: no están vinculados con el cuerpo, sino con el yo psíquico, estos son sentimientos dirigidos por ser aquellos que reaccionan frente a situaciones que se dan en el exterior; y sentimientos espirituales o de la personalidad: estos no surgen del yo, son estados absolutos ya que estos representan un modo de ser y no un modo de estar en el mundo, por lo tanto son permanentes, hacen a la esencia misma de la persona16.

Así mismo el sentimiento de satisfacción es una respuesta emocional y afectiva de la persona a diversos aspectos del trabajo que realiza; en cambio la insatisfacción es una respuesta negativa del trabajador hacia su propio trabajo, de la cual dependerá de las condiciones laborales y la personalidad de cada persona, esto hace referencia al estado de intranquilidad, ansiedad o incluso depresivo que puede llegar una persona que se encuentra insatisfecha laboralmente ${ }^{17}$.

Además la empatía es la capacidad de sintonizar con los sentimientos y las emociones del otro, ponerse en su lugar y pensar qué puede estar sintiendo, de tal manera la persona empática percibe las necesidades y los estados de ánimo de su interlocutor a través de su tono de voz, de su lenguaje postural, sus gestos y también es capaz de reconocer su angustia, sus miedos, aunque no necesariamente ha de tener su mismo punto de vista. También la empatía es la capacidad que posee el profesional de enfermería para pensar y sentir dentro del mundo interno de la persona post operada, le permite sentir la experiencia del otro sin perder la capacidad para evaluar objetivamente los estados mentales de ese otro ${ }^{18}$. Lo que se evidencia en los discursos:

“... Me siento alegre, tranquila y satisfecha por la pronta recuperación del post operado y frente a los cuidados que realizo..." (Azucena)

"...Soy cariñosa y empática en el momento que brindo los cuidados y eso hace que me sienta tranquila y satisfecha porque brindo un cuidado integral..." (Orquídea)

En los discursos, las enfermeras manifiestan como parte de sus experiencias durante su interrelación con las personas post operadas, sentimientos vitales como la tranquilidad y satisfacción, también sentimientos anímicos como la alegría, estos sentimientos son sostenidos en relación directa a la recuperación hemodinámica de la persona post operada, como parte del contagio emocional que generan sentimientos de empatía en la enfermera, ya que durante este periodo, la cuidadora percibe muchos sentimientos, emociones y estados anímicos propios de la experiencia de la intervención quirúrgica, por lo tanto, si la persona tiene una actitud positiva todo se relaciona favorablemente, además el ejercer del cuidado genera sentimientos de satisfacción laboral que es parte de la autorrealización profesional y/o de las compensaciones, lo cual lo lleva a una estabilidad emocional que se ve reflejado en su estado de ánimo e inclusive en sus relaciones interpersonales, estado físico y reiterando positivamente en su calidad de vida como profesional de enfermería.

Así mismo frente al cuidado también se genera la impotencia, siendo un sentimiento desagradable que se debe al desconsuelo de descubrir que de ninguna manera será posible realizar cierta idea, sueño, propósito, meta o modificación; esto se produce cuando sentimos que no hay nada que podamos hacer para reparar un daño o para llevar a cabo cierto objetivo, se sienten completamente 
desconsolado; esta reacción que luego es reproducida como señal de alarma en la situación peligrosa19. En cambio la angustia es un sentimiento universalmente experimentada por el ser humano y está vinculado a situaciones de desesperación cuando el individuo se siente amenazado por algo, donde la característica principal es la perdida de la capacidad de actuar voluntaria y libremente por parte del sujeto ${ }^{20}$. Se evidencia en los discursos:

“... Me siento con impotencia porque hay familiares que no colaboran y no entienden los efectos de la anestesia, entonces esto hace que retrase la recuperación del paciente..." (Margarita)

“...Me angustia y me siento impotente, porque el medicamento no calma el dolor y eso genera mayor ansiedad en el paciente..." (Azucena)

En estos discursos las enfermeras expresan sentimientos negativos que están relacionados con los sentimientos vitales que son la impotencia y angustia, estos se manifiestan en diferentes situaciones, pero la mayoría por la falta de fuerza, poder o competencia para realizar el objetivo; en uno de estos casos se presenta el estado de intranquilidad causado por algo desagradable o amenaza que puede generar sentimiento de culpabilidad cómo se logra evidenciar en lo expresado por las mismas. A pesar de estos motivos siempre hay que tener en cuenta que la unidad de cuidados post anestésicos, es un área crítica y de mucha responsabilidad donde el estado de alerta es una característica o actitud frecuentemente en el equipo de salud que allí labora, aunque hay personas post operadas que se recuperan más rápidos que otros, pero de todas maneras se tiene paciencia para ver su evolución y explicar a la familia, ya que ellos y la persona post operada son indispensables para su recuperación.

\section{B. BRINDANDO UN CUIDADO INTEGRAL A LA PERSONA POST OPERADA}

Regina Waldow afirma que el cuidado es una forma de ser, de expresarse y relacionarse consigo mismo, con otro ser y con el mundo; el cuidado humano envuelve compromiso, protección y responsabilidad, es una forma ética de vivir en la que los seres humanos perciben y reconocen los derechos de los demás, a través del cuidar el ser se humaniza. El proceso de cuidar son todas las actividades desarrolladas por el cuidador para y con el ser cuidado como base en el conocimiento, habilidad, intuición, pensamiento crítico, creatividad, acompañada de comportamientos y actitudes de cuidado para promover, mantener, recuperar la dignidad y totalidad humana. Esta dignidad y totalidad comprende el sentido de integridad y plenitud física, mental, moral, emocional, social, espiritual en las fases de la vida y la muerte constituyéndose en última instancia de un proceso de transformación cuidador y ser cuidado 21.

El personal de enfermería debe estar capacitado y especializado porque en la unidad para el cuidado post operatorio inmediato es un área crítica que abarca desde que la persona sale de quirófano hasta que se estabiliza su estado y se recupera totalmente del estrés de la anestesia y la cirugía. En la Unidad de Cuidados Post anestésica (UCPA) pueden permanecer entre 2 a 4 horas de acuerdo al tipo de anestesia y cirugía, donde se recuperan de los efectos post anestésicos y se brinda el mejor cuidado posible para prevenir complicaciones, es así que el cuidado va dirigido básicamente a asegurar un restablecimiento del equilibrio fisiológico, vigilancia cuidadosa de las funciones vitales que se han visto alterados por el procedimiento quirúrgico y vigilar el efecto de los anestésicos y sedantes ${ }^{3,22}$. 
Así mismo los cuidados de enfermería en el post operatorio inmediato son la conservación de los signos vitales, mantener el estado hemodinámico de la persona, la valoración de las escalas post anestésicas, vigilar el estado de conciencia, vigilar la posición, el control del dolor, administración de medicamentos prescritos conservar la seguridad y promover su comodidad, vigilancia de posibles signos de alerta a nivel cardiovascular, neurológico y gastrointestinal o la aparición de hemorragias y por último e importante registrar el proceso de cuidado en el post operatorio 6 . Cómo se demuestra en los discursos:

“... En pacientes post operado se valora el estado de conciencia y también la escala post anestésica, se realiza el control de signos vitales, la administración de medicamentos y una constante observación de la persona para evitar posibles complicaciones..." (Orquídea)

“... En el post operatorio se realizan cuidados hasta que disminuyan los efectos de la anestesia pero también se tienen en cuenta la parte física, emocional y espiritual de la persona realizando una continua y permanente valoración hemodinámica, manteniendo la comunicación terapéutica y respetando en todo momento su religión..." (Jazmín)

“...No todos los pacientes responden igual a los efectos post anestésicos, algunos tienen escalofríos y les coloco colchas, frezadas para que disminuya el frio y otros hipotensión y se aumenta el goteo del cloruro..." (Azucena)

Según los discursos las enfermeras expresan, que la unidad de cuidados post operatorio inmediato es un área de mucho cuidado y responsabilidad que dura entre 4-8 horas dependiendo de la operación. Lo primero que debe valorar el profesional es el estado de conciencia, control de los signos vitales, observar la herida operatoria y entre otros cuidados; durante el transcurso del tiempo se valora con las escalas de Glasgow, Bromage y Aldrete dependiendo del tipo de anestesia, aunque no todos reaccionan igual a este proceso pero es necesario estar alerta y observar las posibles complicaciones para actuar frente a cualquier complicación por lo tanto las enfermeras de esta área tienen la capacidad y la destreza para poder enfrentar dicho problema.

Además depende de varias circunstancias que pueden cambiar radicalmente su duración, como el tipo de cirugía, la enfermedad que la condicionó, la edad del paciente, su estado general, las enfermedades asociadas y las complicaciones post operatorias si es que las hubo. Por eso la población pediátrica es especialmente susceptible a experimentar un dolor postquirúrgico innecesario por sus limitaciones a la hora de expresar sus sensaciones y necesidades, porque en muchas ocasiones los niños post operados confunden las sensaciones/sentimientos de dolor, ansiedad y de miedo ya que en la mayoría de los infantes no saben expresar bien sus necesidades. En los niños, la valoración del tipo e intensidad de dolor entraña una mayor dificultad y requiere la utilización de escalas de valoración adaptadas a la edad ${ }^{23}$.

El personal de enfermería debe estar familiarizado con el cuidado de niños e idealmente tener un adiestramiento en el manejo de la vía aérea pediátrica, manejo de la fisiología cardiorespiratoria, conocimiento básico de la farmacología de los anestésicos y otros medicamentos; también deben estar familiarizados con el comportamiento propio del desarrollo y las respuestas emocionales de los niños a diferentes edades ${ }^{24}$. Así mismo en la fase postoperatoria la enfermera utiliza la escala de valoración de Steward es un test que valorar la recuperación post operatoria en niños, los parámetros que evalúa son: conciencia, respiración y actividad motora en la cual se emplean para medir el grado de profundidad post 
anestésica hasta los 10 años de edad25, cómo se puede evidencia en los siguientes discursos.

“...Los niños post operados despiertan mareados, asustados, llorando y se desesperan pero la madre apoya y el niño se siente más tranquilo entonces controlo los signos vitales, realizo la valoración post anestésica y administro los medicamentos preescritos..." (Amarillis)

“...Los niños post operados vienen muchas veces irritados, con llanto permanente debido a los efectos post anestésicos, por lo cual lo primero que hago es la valoración según la escala post anestésica, administro el tratamiento indicado, luego hago pasar a la madre y la educo sobre los cuidados y así disminuyo la ansiedad del niño..." (Clavel)

La mayoría de las enfermeras manifiestan que el cuidado en niños es una experiencia muy difícil, ellos como se encuentran frente a personas que desconocen -el equipo de salud- reaccionan con miedo y llanto. En este contexto, gracias a la relación paciente- madre- enfermera al niño se le brinda confianza y así se puede realizar cuidados integrales.

\section{RESPONSABILIDAD VS TEMOR E} IMPOTENCIA FRENTE A LAS COMPLICACIONES POST OPERATORIAS

El temor y la impotencia son sensaciones desagradables, una de ellas surge por la aversión natural a las amenazas porque al sentir temor la persona responde con rapidez ante una situación adversa, en cambio la impotencia es por la falta de poder hacer algo ante una situación, pero la responsabilidad es una forma de actuar que se adquiere a través de la experiencia y la práctica, ya que la persona se compromete a responder $y$ tomar decisiones de sus actos. Además la responsabilidad nos da estabilidad de lo que se quiere conseguir; por ello la enfermera debe aceptar y reconocer su responsabilidad individual que le compete en la toma de decisiones durante su desempeño profesional en los diferentes ámbitos y durante el cuidado20, 26 .

Waldow, durante el proceso de cuidado sostiene que, la cuidadora deberá poner en práctica su habilidad de pensamiento crítico, con la reflexión siempre presente sobre lo que está sucediendo, está realizando y cómo deberá comportarse; en cada nuevo encuentro deberá evaluar el estado de la persona post operada, observándolo como un todo. El cuidar de enfermería incluye los comportamientos y actitudes que se demuestran en las acciones de enfermería que le son pertinentes, traducidos en conocimientos, habilidades manuales, creatividad, sensibilidad, pensamiento crítico, capacidad de decisión, consideración y respeto, para poder reducir o evitar posibles complicaciones ${ }^{21}$.

Durante la fase del post operatorio inmediato, en esta área el profesional de enfermería está capacitado buscando principalmente asegurar un restablecimiento del equilibrio fisiológico de la persona y estar alerta frente a cualquier eventualidad para actuar de manera rápida, en este periodo muchas veces la persona esta inconsciente pero, de igual manera el cuidado va dirigido a brindar las medidas de seguridad para evitar complicaciones; por eso la enfermera es de vital importancia para la detección y abordaje precoz de las complicaciones en el post operatorio inmediato $^{3}$. El rol de enfermería en el ámbito asistencial engloba aquellas actuaciones incluidas en el campo de conocimiento enfermero y por lo tanto el profesional está capacitado para decidir, actuar y evaluar resultados de manera autónoma; en cuanto a posibles complicaciones la actuación se lleva a cabo de forma conjunta con el equipo médico y la vigilancia27. 
El enfermero(a) de la unidad de recuperación, es un profesional humano, dinámico, amable, analítico, eficiente, responsable, en permanente actualización y gestor de cambios, que ha adquirido competencias científico-técnicas para brindar cuidado post operatorio inmediato $y$ ayudar a la persona quirúrgica. Durante la recuperación de la persona post operada la coordinación del equipo es fundamental para la estabilización del usuario en el menor tiempo posible. Por tanto, el post operatorio se realiza la valoración y detección precoz de posibles complicaciones, y la cobertura de necesidades básicas ${ }^{28}$.

En el trabajo diario, enfermería está expuesta a situaciones desagradables y molestas; sin embargo el enfermero tiene que modificar o suprimir sus propios sentimientos para conseguir que las personas se sientan cuidadas y seguras, independientemente de lo que él sienta. Es entonces cuando se produce una diferencia entre las emociones sentidas y las expuestas al exterior, lo que implica un gasto extra de fuerza y energía, llamado trabajo emocional, de manera que mientras más intensa sea la emoción a la que se tienen que enfrentar, más duro será el trabajo emocional que se realice ${ }^{29}$. La enfermera lo expresa en los discursos:

“...Hay post operados que la presión baja demasiado o aumenta pero igual monitorizo continuamente, realizo acciones de enfermería y comunico al médico de turno..." (Azucena)

"...Me siento impotente de no poder resolver complicaciones pero igual monitoreo continuamente hasta que sea evaluado $y$ trasladado a un hospital de mayor complejidad..." (Clavel)

Las enfermeras expresan que el post operatorio inmediato es un área crítica y de mucha responsabilidad donde tienen que estar alerta para reaccionar frente a cualquier complicación, porque en instante puede alterarse alguna función; con la capacidad y destreza que se identifican en esta área, se puede solucionar, aunque frente a estos problemas se muestran sentimientos de impotencia y temor pero hay que saberlos llevar, no ser confiadas porque hay personas post operadas que se recuperan más rápidos que otros, por eso la enfermera observa minuciosamente, monitoriza y conversa con cada persona para ver cómo está evolucionando, cómo se encuentra en esos momentos y poder prevenir complicaciones. Ya que la enfermera realiza acciones frente a la situación que se encuentra la persona, al mismo tiempo comunica al médico de turno y al no solucionarlo el equipo multidisciplinario deciden trasladar a un hospital de mayor complejidad.

\section{Conclusiones}

1. Dentro de las experiencias de las enfermeras en el cuidado del hospital Naylamp, en el servicio de la unidad de cuidados post anestésicos se evidencia que afloran sentimientos personales ante la situación que atraviesa la persona post operada, que surge por un contagio emocional en respuesta a las experiencias de brindar cuidados, generando sentimientos de satisfacción e insatisfacción de la enfermera.

2. Las enfermeras reconocen lo importante de brindar cuidado integral en la parte física, psicológica, emocional y espiritual ante una situación además de mantener una interacción continua con la persona post operada involucrando a la familia mucho más a la del niño post operado, esto accede que las personas expresen y compartan emociones, sentimientos y necesidades en la cual permite establecer una relación de confianza y lograr un cuidado óptimo. 
3. Las enfermeras de la unidad de cuidados post anestésicos expresan temor, miedo e impotencia frente a complicaciones post operatorias que les lleva a reflexionar asertivamente y asumir con responsabilidad y compromiso las decisiones en el cuidado, además siendo un área de especialidad exigen que los profesionales que trabajan en esa área mantengan a una preparación continua, además la enfermera por el actuar que realiza crece en forma personal y profesional.

\section{Bibliografía}

1. Du Gas B. Tratado de Enfermería Práctica. 4a ed. México: McGraw- Hill Interamericana; 2000.

2. American Nurses Association [Sede web] 2013 [Acceso 08 de Abril de 2014]. Disponible en: http://www.suagm.edu/umet/biblioteca/Reserva_ Profesores/reina_rivero_nurs_105/Definiciones_e nfermeria.pdf

3. Brunner L, Suddarth D. Enfermería Médico Quirúrgica. McGraw-Hill Interamericana. México. 2002.

4. Long B, Phipps W, Cassmeyer V. Enfermería médico quirúrgica: Un enfoque del proceso de enfermería. 3a ed. Madrid: HarcourtBrace; 1998.

5.Burga S, Cáceres C, Elorreaga O. Experiencias de los pacientes frente al cuidado de enfermería en el alivio del dolor post operatorio inmediato, servicio de cirugía del Hospital Provincial Docente Belén. [Tesis licenciado]. Lambayeque: Universidad Nacional Pedro Ruiz Gallo; 2005.

6. Salvatierra V, Rivera C. Gestión del cuidado esencia de ser enfermera. [Sede Web] Perú; 2014. [Acceso 28 de mayo de 2015]. Disponible en: $\quad$ http://www.cies.cl/Gestion-del\%20Cuidado.pdf

7. Carrillo E. Estándar de Proceso para el Cuidado de Enfermería a pacientes quirúrgicos en el post- operatorio inmediato en la Unidad de Cuidados Post-Anestésicos del Hospital Dr. Ricardo Baquero González. Primer Trimestre año 2005. [Tesis licenciado]. Caracas: Universidad Central de Venezuela; Mayo 2005. [Acceso 19 de mayo de 2015]. Disponible en:

http://saber.ucv.ve/jspui/bitstream/123456789/ 452/1/Mrie\%20Guerrero\%20y\%20Carla\%20Romer o.pdf

8. Sánchez S. Max Scheler: Idea de la fenomenología. Philosophica: Enciclopedia filosófica on line [revista on line] 2007 [Acceso 29 de mayo de 2015]. Disponible en: http://www.philosophica.info/voces/scheler/Sche ler.html

9. Soza A. Experiencia de las estudiantes de enfermería en el cuidado de personas en agonía de la Universidad Santo Toribio de Mogrovejo [Tesis licenciado]. Chiclayo: Universidad Santo Toribio de Mogrovejo. Escuela de Enfermería; 2009.

10. Mejía J. El muestreo en la investigación cualitativa. Investigaciones sociales [Revista en Internet] 2000 [Acceso 23 de junio de 2015]; vol.7 (5).

Disponible en: http://sisbib.unmsm.edu.pe/bibvirtualdata/publi caciones/inv_sociales/n5_2000/a08.pdf

11. Palacios D, Corral I. Fundamento y Desarrollo en un protocolo de investigación fenomenológica en enfermería. Rev Enfermería intensiva [Revista en internet] 2010. [Acceso 26 de mayo de 2014]. 21(2). Disponible en:

http://seeiuc.org/revista.html?start $=15$

12. Sgreccia E. Manual de Bioética: fundamento y ética biomédica. 2 da ed. Madrid, Editorial: Albijes S. L; 2007.

13. Hernández S. Metodología de la investigación. 5 ta ed. México: McGraw-Hill/Interamericana Editoriales; 2010. 
14. Wojtyla K. Persona y Acción. 1 era ed. Madrid: Editorial Católica; 2005.

15. Castillo del Pino C. Teoría de los sentimientos. [Libro en internet]. Barcelona: Tusquets Editores S.A; 2005. [Acceso 20 de junio de 2015]. Disponible en: https://books.google.es/books?isbn $=848310708$ $\underline{2}$

16. Fernández A. Fundamentos de la psiquiatría actual. [Libro en internet]. Madrid; 1979. (p 264266). [Acceso 27 de mayo de 2015]. Disponible en:

http://www.um.edu.ar/catedras/claroline/backen ds/download.php?url=LONsYXNpZmljYWNpb25fZ GVfbG9zX3NIbnRpbWIlbnRvcy5kb2M\%3D\&cidRese $\underline{\mathrm{t}=\text { true } \& \text { cidReq }=\mathrm{M} 12}$

17. Buelga M. Satisfacción e Insatisfacción: Clima Laboral. Gestiopolis [Revista en Internet] 2011. [Acceso 27 de mayo de 2015]. Disponible en: http://www.gestiopolis.com/satisfaccioninsatisfaccion-clima-laboral/

18. Sánchez J. Empatía y desgaste profesional en enfermeras del área de salud mental y psiquiátrica. Revista Portales Médicos [Revista en internet] 2009. [Acceso 27 de mayo de 2015]. Disponible en:

http://www.portalesmedicos.com/publicaciones/ articles/1771/5/Empatia-y-desgasteprofesional-en-enfermeras-del-area-de-saludmental-y-psiquiatrica.

19. Ruiz M. Frustración e impotencia [Sede web]* 2015. [Acceso 13 de mayo de 2015]. Disponible en:

http://www.oratorianet.com/rsp/rsp3/COMENTA RIO46.html

20. Carlos J, Ortega V, Zubeidat I. Ansiedad, angustia y estrés: tres conceptos a diferenciar. Revista Mal-Estar E Subjetividade [Revista en internet]* 2003 Marzo. [Acceso 13 de mayo de
2015]; Nº 1. Disponible

en:

http://pepsic.bvsalud.org/pdf/malestar/v3n1/02. pdf

21. Waldow R. El cuidado integral del ser humano. En: Memorias del III Congreso Internacional y V Nacional de Enfermería en Cuidado Humano. [Libro en internet]. Venezuela: Universidad de Carabobo; 2013. (p. 53-62). [Acceso 19 de mayo de 2014]. Disponible en:

http://apps.elsevier.es/watermark/ctl_servlet?_f= $10 \&$ pident_articulo $=90367024 \&$ pident_usuario $=0$ \&pcontactid $=\&$ pident_revista $=400 \&$ ty $=22 \&$ accion $=$ L\&origen $=$ zonadelectura\&web $=w w w$.elsevier.es $\& l a n=e s \& f i c h e r o=400 v 11$ n04a90367024pdf001. pdf

22. García J, Gil E, Giménez A, Giménez D et al. Enfermería Médico quirúrgica. [Libro en internet]. Madrid: Mosby; 2004. [Acceso 28 de mayo de 2015]. Disponible en: http://mural.uv.es/rasainz/1.1_GRUPO3_POSTOP ERATORIO.pdf

23. Pérez I, Bellido A. Dolor y Ansiedad en Cirugía pediátrica. Secipe [Revista en Internet] 2008. [Acceso 28 de mayo de 2015]; vol.21 (2).Disponible en:

http://www.secipe.org/coldata/upload/revista/21 207.pdf

24. Guillamet A, Jerez J. Enfermería Quirúrgica: Planes de cuidados. 3era ed. Barcelona: SpringerVerlag Ibérica; 1999.

25. Rebecca J. Entendiendo Anestesia Pediátrica. [Libro en internet]. New Delhi: Confederación Latinoamericana de Sociedades de Anestesiología; 2008. [Acceso 28 de mayo de 2015]. Disponible en:

http://www.clasaanestesia.org/site/version/docs Llibro_ap/chp-13.pdf

26. Jiménez E, Gómez M. Maneras de cuidar de la enfermera a la persona hospitalizada desde la 
perspectiva del estudiante. Unitru [Revista en Internet] 2013 Julio-Diciembre. [Acceso 18 de mayo de 2015]; vol. 11(2). Disponible en: revistas.unitru.edu.pe/index.php/facenf/issue/do wnload/87/131

27. Torres M, Buil G, Perez J, Sandino M. Cuidados de enfermería según el grado de hematuria relacionado con los gramos de próstata resecados. Asociación Española de enfermería en Urología [Revista en Internet] 2010. [Acceso 13 de mayo de 2015]; N 115. Disponible en:

http://dialnet.unirioja.es/servlet/revista?codigo $=$ $\underline{13453}$

28. Mendoza J, Narváez R. Cuidados de enfermería en la prevención de complicaciones cardiopulmonares durante la fase postoperatoria en pacientes hospitalizados en la clínica docente "Los jarales". [Tesis licenciado]. Venezuela:
Universidad Central de Venezuela; 2012. [Acceso 19 de mayo de 2015]. Disponible en:

http://saber.ucv.ve/jspui/bitstream/123456789/ 8605/1/Tesis\%20EE2013\%20M523\%20J4.\%20CUI DADOS\%20DE\%20ENFERMER\%C3\%8DA\%20EN\%20L A\%20PREVENCI\%C3\%93N\%20DE\%20COMPLICACIO NES\%20CARDIOPULMONARES\%20DURANTE\%20LA \%20FASE\%20POSTOPERATORIA\%20EN\%20PACIENT ES\%20HOSPITALIZADOS\%

29. López S. Implicación emocional en la práctica de la enfermería. Cultura de los cuidados: Revista de enfermería y humanidades [Revista en Internet] 2000. [Acceso 27 de mayo de 2015]; (7):172-180. Disponible en:

http://rua.ua.es/dspace/bitstream/10045/5120/ 1/CC_07-08_20.pdf. 